Research Paper

\title{
The effects of fluoride based fire-fighting foams on soil microbiota activity and plant growth during natural attenuation of perfluorinated compounds
}

\author{
Renato Nallin Montagnolli ${ }^{a}$, Paulo Renato Matos Lopes ${ }^{\mathrm{b}}$, Jaqueline Matos Cruz ${ }^{\mathrm{a}}$, \\ Elis Marina Turini Claro ${ }^{\mathrm{a}}$, Gabriela Mercuri Quiterio ${ }^{\mathrm{a}}$, Ederio Dino Bidoia ${ }^{\mathrm{a}, *}$ \\ a Departamento de Bioquímica e Microbiologia, Instituto de Biociências, UNESP - São Paulo State University, Avenida 24 A, 1515-Bela Vista, 13506-900, Rio \\ Claro-SP, Brazil \\ b Faculdade de Ciências Agrárias e Tecnológicas, UNESP - São Paulo State University, Rodovia Comandante João Ribeiro de Barros (SP 294), Km 651, \\ 17900-000, Dracena-SP, Brazil
}

\section{A R T I C L E I N F O}

\section{Article history:}

Received 1 November 2016

Received in revised form

27 December 2016

Accepted 26 January 2017

Available online 30 January 2017

\section{Keywords:}

AFFF

Perluorinated compounds

Germination

Response surface analysis

Soil toxicity

\begin{abstract}
A B S T R A C T
The use of fluoride based foams increases the effectiveness of fire-fighting operations, but they are also accompanied by major drawbacks regarding environmental safety of perfluorinated compounds (PFCs). The main concern with PFCs release is due to their well-known persistence and bioaccumulative potential, as they have been detected in many environmental samples. There is a significant knowledge gap on PFC toxicity to plants, even though such data could be useful towards bioremediation procedures. It is consensus that a realistic assessment of fire-fighting foam toxicity should cover as many test organisms as possible, however, few studies combine the performance of ecotoxicological tests with a detailed study of microbial communities in soil contaminated with firefighting foams. Our research evaluated the effects of natural attenuation of PFCs on the development of arugula and lettuce seeds. The effects of variable PFCs amounts were also observed in soil microbiota using the 2,6 dichlorophenol-indophenol redox dye as microbial metabolism indicator. We aimed to determine whether aqueous film forming foams toxicity increased or decreased over time in a simulated contamination scenario. We argued that the long-term biotransformation of fire-fighting foams should be taken in to account when evaluating toxicity, focusing on a time-based monitoring analysis, since potentially toxic intermediates may be formed though biodegradation. The phyto-toxicity of PFCs to lettuce and arugula was high, increasing as a function of the concentration and decreasing as a function of exposure time to the environment. However, very specific concentrations throughout biodegradation result in the formation of non-inhibiting intermediates. Therefore, variable biodegradation-dependent germination rates may be misleading on non-time-based monitoring approaches. Also, the low phyto-toxicity after 240 days does not exclude the potential for PFC bioaccumulation in plants. We also proposed that the colorimetric data modelling could also establish a novel toxicity parameter to evaluate the release impacts to soil and biota. The combined assays allowed the monitoring of PFCs during long-term exposition to plants as well as their immediate effects on the same soil microbiota.
\end{abstract}

(C) 2017 Elsevier B.V. All rights reserved.

\section{Introduction}

Firefighting foams have been developed for better adhesion to materials on fire, producing a continuous coating on it. Their low density allows better spreadability over the surface of burning materials, covering and isolating them from atmospheric oxygen.

\footnotetext{
* Corresponding author.

E-mail address: ederio@rc.unesp.br (E.D. Bidoia).
}

The suppression of oxygen and the cooling of burning materials prevent re-ignition. The use of fluorine-containing aqueous film forming foams (AFFF) has improved the firefighting effectiveness of hydrocarbon related operations (Sardqvist, 2002) aiming to ensure the promptness and safety of firefighting techniques. The AFFF advantages are clear, but they are also accompanied by major drawbacks concerning environmental safety.

Firefighting foams contain various substances to achieve proper formation of foam and grant its functional properties. Most of the foam compounds are fluorinated surfactants or hydrolyzed 
proteins, solvents and water. Commercial formulations of AFFF are complex mixtures whose main components are a solvent (typically a glycol ether), fluorinated surfactants (amphoteric or anionic partially fluorinated or perfluorinated), and surfactantbased hydrocarbons. Fluorinated surfactants in AFFF contribute to its performance when extinguishing fires (Kissa, 1994; Alm and Stern, 1992; Falk, 1978). The presence of fluorine contributes to the rigidity of perfluorocarbon chains (Key et al., 1997). The fluoro-carbon bond is strongly polarized. Fluorination also reinforces C-C adjacent bonds (Hudlicky and Pavlath, 1995). Therefore, perfluorinated surfactants are more thermally stable than their corresponding hydrocarbon analogs. In particular, perfluorinated carboxylic acids (PFCAs) and perfluorinated sulfonic acids (PFSAs), both found in AFFF, are among the most thermally stable perfluorinated compound ( $\mathrm{PFC}$ ) groups. In addition to thermal stability, perfluorinated surfactants are stable to acids, bases, oxidizers and reducers. This stability allows fluorinated compounds to remain intact in environments where hydrocarbon surfactants are degraded (Kissa, 1994).

The main concern with AFFF release is due to PFCs persistence and their bioaccumulative potential. They have been detected in many environmental samples, including air, surface water (Murakami et al., 2008; Kim and Kannan, 2007), waste waters (Sinclair and Kannan, 2006), soil (Higgins et al., 2005) and groundwater (Schultz et al., 2004). PFCs were also found accumulating in biota, including mammals (Giesy and Kannan, 2002) and humans (Hölzer et al., 2008). The PFCAs, PFSAs and their potential precursors have attracted attention as global contaminants (Buck et al., 2011). PFCAs and long chain PFSAs are described as very problematic because they are highly persistent (Frömel and Knepper, 2010), bioaccumulative and found scattered almost universally in abiotic environments (Rayne and Forest, 2009), in biota (Giesy and Kannan, 2001), food (Clarke and Smith, 2011) and humans (Vestergren and Cousins, 2009). As a result, many firefighting foams based on PFCs had their production restricted and were listed as substances of very high concern in European Regulation of Chemicals (ECHA, 2013).

The AFFFs are predominantly released in the form of liquid foam, which increases the potential of PFAS to penetrate in aquatic environments. The PFC inflow to the medium may occur via four routes: (i) release of volatile PFCs into the atmosphere (Dinglasan-Panlilio and Mabury, 2006), which is oxidized photochemically (Ellis et al., 2004) and back to the water cycle by precipitation; (ii) discharge to wastewater treatment plants (Yu et al., 2009); (iii) discharge of urban runoff contaminated by diffuse sources (Houtz and Sedlak, 2012; Zushi and Masunaga, 2009), and (iv) the infiltration of waste and spills in groundwater (Moody and Field, 2000; Moody et al., 2003). Even though the PFC abiotic routes have been continuously investigated, there is still a lack of knowledge about the PFCs from the toxicological standpoint in many organisms.

Toxicity data can be used to better remediate AFFF contaminated areas. Toxicity tests are based on determining the potential impact of pollutants towards biota in a set environment (Hagner et al., 2010). Thus, ecotoxicological datasets have long been used with relative success as an additional tool for monitoring the efficiency of soil bioremediation, making it essential to assess environmental hazards in contamination scenarios (Lladó et al., 2012; Sheppard et al., 2011). However, few studies combine the performance of ecotoxicological tests with a detailed study of microbial communities in soil contaminated with firefighting foams.

It is consensus that a realistic assessment of AFFF toxicity should cover as many test organisms as possible. Phyto-toxicity tests with AFFF sources are scarce in current the literature. We also argue that the biotransformation of AFFF compounds should be taken in to account when evaluating long-term toxicity, as potentially toxic intermediates may be formed. Our research evaluated the effects of natural attenuation of AFFF on the development of plants. We aimed to determine whether AFFF toxicity increases or decrease over time in a simulated soil contamination scenario. Unlike most studies found in the literature, which monitor AFFF original formulation or persistent final biotransformation products, we designed intermediate toxicity evaluation points. Changes that affected the development of vegetable tissues provided an overall assessment of PFCs environmental safety. Moreover, we aimed to propose a novel toxicity classification towards environmentally safe release of pollutants using a colorimetric approach. A redox indicator $(2,6$ dichlorophenol-indophenol) usually associated with biodegradation studies was repurposed by our research group to evaluate soil microbiota response to various concentrations of AFFF.

\section{Material and methods}

\subsection{Soil samples}

Soil samples were acquired from the Biosciences Institute Experimental Garden at the Sao Paulo State University in Rio Claro, SP, Brazil $\left(22^{\circ} 43^{\prime} 24.2^{\prime \prime} \mathrm{S} 47^{\circ} 08^{\prime} 00.3^{\prime \prime} \mathrm{W}\right)$. The area has a petroleum contamination background that is analogous to oil industry sites affected by hydrocarbon fires. The sampling area has been exclusively used for experiments with gasoline, diesel, kerosene and other petroleum hydrocarbons over the past 8 years.

\subsection{Toxicity assessment of AFFF dilutions}

Various dilutions of AFFF samples were prepared from stock AFFF solution. In this first group of experiments no soil was added to the assays. We evaluated pure AFFF effects on plants germination, wherein the concentration of perfluorinated compounds was $195 \mathrm{~g} \mathrm{~L}^{-1}$. Dilutions were then made to match $3 \%$ and $1 \%$ concentrations, yielding 97.5 and $19.5 \mathrm{~g} \mathrm{~L}^{-1}$ of PFCs. Both dilutions are also available from firefighting foam distributors. The solutions were then directly inserted into toxicity bioassays for the germination and development of seeds.

\subsection{Toxicity throughout biodegradation}

A set of experiments on toxicity was performed to evaluate AFFF toxicity at different concentrations through natural attenuation. The soil matrix was designed to simulate widespread PFC contamination scenarios with AFFF. The biodegradation environment was set up through a simulated soil contamination within a plastic bag filled with $3 \mathrm{~kg}$ of soil and $0.1 \mathrm{x}, 1 \mathrm{x}$ and $10 \mathrm{x} \mathrm{m} / \mathrm{v}$ AFFF (Table 1 ). The container had small holes with approximately $1 \mathrm{~mm}$ diameter, spaced $1 \mathrm{~cm}$ each, to promote the exchange of microorganisms between the inner soil and the external environment. The recipient was buried $5 \mathrm{~cm}$ from the surface. We used the retail 6\% AFFF (Sintex S1371/11) formulation, commercially available and commonly applied to petroleum fires in Brazil. Its formulation contains

Table 1

Phyto-toxicity bioassays.

\begin{tabular}{llll}
\hline Sample ID & Components & AFFF Concentration & Biodegradation time \\
\hline C0 & Soil & - & 0 days \\
C60 & Soil & - & 60 days \\
C120 & Soil & - & 120 days \\
C180 & Soil & - & 180 days \\
C240 & Soil & - & 240 days \\
F0 & Soil + AFFF & $0.1 \times, 1 \times$ and $10 \times \mathrm{m} / \mathrm{v}$ & 0 days \\
F60 & Soil + AFFF & $0.1 \mathrm{x}, 1 \mathrm{x}$ and $10 \times \mathrm{xm} / \mathrm{v}$ & 60 days \\
F120 & Soil + AFFF & $0.1 \mathrm{x}, 1 \mathrm{x}$ and $10 \times \mathrm{xm} / \mathrm{v}$ & 120 days \\
F180 & Soil + AFFF & $0.1 \mathrm{x}, 1 \mathrm{x}$ and $10 \times \mathrm{xm} / \mathrm{v}$ & 180 days \\
F240 & Soil + AFFF & $0.1 \mathrm{x}, 1 \mathrm{x}$ and $10 \mathrm{xm} / \mathrm{v}$ & 240 days \\
\hline
\end{tabular}


perfluorinated substances fluorotelomer thioether amido sulfonate (FtTAoS) known by the brand name Lodyne, and diethyleneglycol butyl ether (DGBE or butyl carbitol). Together, these substances constitute the largest part of the concentrated formulation of Sintex AFFF.

The treatment groups were removed at 60,120, 180 and 240 days. Once collected, the soils were stored at $4.0 \pm 0.1^{\circ} \mathrm{C}$ before further toxicological analysis.

The AFFF added to each plastic container matched the concentrations from recently reported spills in soil (USEPA, 2009a,b). The concentrations were adjusted to better represent an oil refinery after the application of firefighting foam. The typical concentration of AFFF found in soil is up to $15.0 \mathrm{gL}^{-1}$ soil (USEPA, 2009a,b), resulting in $4.5 \mathrm{~mL} \mathrm{~kg}^{-1}$, considering the average soil density as $1.5 \mathrm{~g} \mathrm{~cm}^{3}$ and AFFF as $1.12 \mathrm{~g} \mathrm{~cm}^{-3}$. The amount of AFFF in the simulated soil contamination was conducted in 3 orders of magnitude, namely: $0.1 \mathrm{x}$ ( or $\left.0.45 \mathrm{~mL} \mathrm{~kg}^{-1}\right), 1 \mathrm{x}\left(4.5 \mathrm{~mL} \mathrm{~kg}^{-1}\right)$ and $10 \mathrm{x}\left(45 \mathrm{~mL} \mathrm{~kg}^{-1}\right)$. Each treatment was buried back at the Experimental Garden of the Institute of Biosciences - Sao Paulo State University, Rio Claro - SP. The soil samples were dug up and had their phyto-toxicity analyzed after 60, 120, 180 and 240 days.

\subsubsection{Phyto-toxicity bioassays}

From a physiological point of view, germination occurs when the embryo ceases physiological hiatus and goes into metabolic activity (Nassif et al., 1998), provided that internal and environmental conditions are met for the tissues to develop. Any changes in these parameters by toxic substances may affect the development of plant tissue (Ayers and Westcot, 1999). Based on this, our toxicity assays were performed in soils according to previous protocols proposed by Morales (2004) and Montagnolli et al. (2015). The effects of AFFF were evaluated using two test organisms: Lactuca sativa (lettuce) and Eruca sativa (arugula).

The pesticide-free seeds were all purchased from the same supplier (TopSeed $^{\circledR}$ - blue line). Each bioassay was performed in triplicate per soil sample. Positive controls with pure MilliQ water and negative controls with $\mathrm{ZnSO} 40.10 \mathrm{M}$ were performed to verify seed sensitivity and viability.

Seed germination and seedling tissue elongation was measured. Germination tests were conducted in petri dishes containing a filter paper that covered its entire base. We added $4.0 \mathrm{~mL}$ of soil leachate from each assay at the filter papers. In other words, we poured into each filter paper the supernatant portion of each soil bioassay proposed in Table 1 . The leachate source was kept under agitation at $210 \mathrm{rpm}$ in shaker table for $24 \mathrm{~h}$. Each vial contained $5.0 \mathrm{~g}$ of soil and $100.0 \mathrm{~mL}$ of deionized water. The seeds were then placed on top of the filter paper. The bioassays were performed with lettuce and arugula separately, as 30 seeds were added to individual petri dishes. Experiments were conducted in triplicate for each test substance. The petri dishes were covered with plastic film to keep moisture, and incubated at $20.0 \pm 1.0^{\circ} \mathrm{C}$ for $120 \mathrm{~h}$ under dark conditions.

\subsubsection{Germination and growth analysis}

Our analysis of seedling development started by freezing seedlings after $120 \mathrm{~h}$ of incubation. This procedure ceased vegetable growth and decreased the rigidity of plant tissues to facilitate measurements by avoiding possible breaches during experimental handling. Root and hypocotyl measurements were taken for each seedling. Germination count was performed after $120 \mathrm{~h}$. Seeds were considered germinated from a minimum $2 \mathrm{~mm}$ radicle protrusion limit. The data obtained in the tests were used to calculate the germination percentage (\% G) given by Labouriau and Agudo (1987) according to Eq. (1).

$\% \mathrm{G}=\arcsin [(\mathrm{SGa} / \mathrm{SGt}) \cdot 100]^{0.5}$ where: SGa is the total number of germinated seeds and SGt is the total number of germinated control seeds;

The statistical analysis verified the difference between treatments by using the Tukey test at 5\% probability (Sokal and Rohlf, 1995). Germination values were normalized according to legacy references by Snedecor (1962) to allow the comparison across our all of our results.

\subsubsection{Data plotting and interpretation}

A novel approach towards germination data analysis was performed using a three-dimensional response surface relating germination time and concentration of AFFF on an axis $\mathrm{x}, \mathrm{y}$ and $z$. This data plotting technique evaluates the influence of two factors (time and concentration) simultaneously in the germination of arugula and lettuce seeds. The 3D plots were made with MICROCAL ORIGIN 8.0 software. It is important to highlight that the experiments have not been based on factorial experimental design and, unlike factorial design, the color gradient in our study is filled by linear extrapolation of the experimental data directly through a multidirectional method proposed by Renka (1984) and Yuan (1998). Therefore, the response surface was created from the raw data converted into a three-dimensional Cartesian coordinate plane using Eq. (2) system.

$$
\begin{aligned}
& \mathrm{r}=\left(\mathrm{x}^{2}+\mathrm{y}^{2}\right)^{1 / 2}(\mathrm{a}) \\
& \theta=\tan ^{-1(\mathrm{y} / \mathrm{x})}(\mathrm{b}) \\
& \mathrm{z}=\mathrm{z}(\mathrm{c})
\end{aligned}
$$

Each variable in Eq. (2) - time (x), AFFF concentration (y) and germination $(z)$ - went through the process of triangulation and definition of response surface contours. The first step was to create a three-dimensional map and triangulation of data based on the distance between each. All points of data were connected to create triangles in a plane $x$ and $y$. Each of these points had a value of $z$. The angles were designed to be as close as possible to equilateral geometry. Moreover, the triangles could not intersect. The layers were set perpendicularly through all planes of the $z$ axis looking for the sides of the triangles of the three-dimensional map. Where the side of each triangle intersects the plane, a point was defined. Each of these points on separate planes were computed by linear interpolation to generate the polygons with a contour line of the response surface (xc, yc, zc). In other words, the contour line is drawn at the edge of the triangles that form the three-dimensional data map.

The process of searching for intersection points of threedimensional triangulation map was repeated in several planes for creating various contour lines. The outline was not smoothed with spline type curves since it would result in less data precision on intermediate values. The resolution of the response surface was given by the number of contour levels made on the plan of triangulation of data. Each of the planes was assigned to a different shade of gray. In this study, we described the germination data with 50 distinct levels, each spanning $2 \%$ units.

\subsection{Redox indicators and microbial inhibition}

The colorimetric approach towards toxicity is fundamentally different from the described phyto-toxicity assays, as they are a short-term detection protocol. Colorimetric methods are often referred to as rapid and low cost procedures in detecting microbial metabolism occurrence. Yet, both methods can provide equivalent data in toxicity processes. Generally, colorimetric methods are based in the different coloration of oxidized and reduced forms. Due to this fact, such color change can be monitored with a spectrophotometer, evaluating changes in redox indicators absorbance values in a set time or across the discoloration process. 
Table 2

Colorimetric bioassays.

\begin{tabular}{lllll}
\hline Assay ID & DCPIP & BH Media & Soil Microbiota & AFFF \\
\hline C1 & $400 \mu \mathrm{L}$ & $7,5 \mathrm{~mL}$ & - & - \\
C2 & $400 \mu \mathrm{L}$ & $7,5 \mathrm{~mL}$ & $100 \mu \mathrm{L}$ & - \\
C3 & $400 \mu \mathrm{L}$ & $7,5 \mathrm{~mL}$ & - & $1000 \mu \mathrm{L}$ \\
B1 & $400 \mu \mathrm{L}$ & $7,5 \mathrm{~mL}$ & $100 \mu \mathrm{L}$ & $1000 \mu \mathrm{L}$ \\
B2 & $400 \mu \mathrm{L}$ & $7,5 \mathrm{~mL}$ & $100 \mu \mathrm{L}$ & $100 \mu \mathrm{L}$ \\
B3 & $400 \mu \mathrm{L}$ & $7,5 \mathrm{~mL}$ & $100 \mu \mathrm{L}$ & $10 \mu \mathrm{L}$ \\
\hline
\end{tabular}

The 2,6 dichlorophenol-indophenol (DCPIP) is an enzymecatalyzed redox electron acceptor that is blue in its oxidized form $\left(\mathrm{DCPIP}_{\mathrm{ox}}\right.$ ) and colorless in its reduced form (DCPIP ${ }_{\text {red }}$. DCPIP $\mathrm{Dx}_{\mathrm{ox}}$ loss of color is monitored at a wavelength of $600 \mathrm{~nm}$ since its peak absorbance occurs at $600 \mathrm{~nm}$, as reported by Yoshida et al. (2001). By incorporating an electron acceptor such as DCPIP ${ }_{\text {ox }}$, it was possible to ascertain the ability of a microorganism to grow on AFFF dilutions by simply observing the color change. The redox reactions applied to AFFF allowed a brief, yet responsive analysis of toxicity in various concentrations.

To study AFFF toxicity to the soil microbiota, colorimetric assays were setup using $5 \mathrm{~g}$ of $\mathrm{CO}$ soil (Table 1 ) aliquot suspended in $100 \mathrm{~mL}$ of Bushnell-Haas (BH) media (Montagnolli et al., 2015). Initial experiments optimized the DCPIP ${ }_{\mathrm{ox}}$ color change output in bioassays by adjusting AFFF initial concentration, soil microcosm cell density and length of incubation with DCPIP ${ }_{\text {ox }}$. After determining ideal substrate concentration, the cells were counted using standard Plate Count Agar and transferred from storage culture.

The microbial cultures were reactivated and inoculated to $50 \mathrm{~mL}$ of BH medium (Montagnolli et al., 2015) at $35^{\circ} \mathrm{C}$ for biomass growth along with AFFF. No agitation or darkness conditions were applied. After $48 \mathrm{~h}$ in $\mathrm{BH}$ medium, the $\mathrm{CO}$ soil culture was inoculated to tubes along with DCPIP ${ }_{\text {ox }}$ indicator and AFFF. Colorimetric assays were conducted in test tubes with lids, to retain $\mathrm{CO}_{2}$ saturation. The tubes were transparent and suitable for spectrophotometry measurements. The contents of each colorimetric assay followed the concentrations in Table 2. All assays were performed in triplicates.

After assembled, each tube underwent vortex agitation for $10 \mathrm{~s}$ and were then stored at $35^{\circ} \mathrm{C}$ and $180 \mathrm{rpm}$. Control assays $\mathrm{C} 1$ and C2 (media control and bacterial control) evaluated interactions of the assay components and DCPIP ${ }_{\text {ox }}$ indicator. A third control set (C3 - AFFF control) verified the sole AFFF influence in DCPIP discoloration. The full assays included DCPIP ${ }_{\text {ox }}$ indicator, $\mathrm{BH}$ medium, soil inoculum and the different AFFF concentrations. The absorbance of all assays was measured by a $\mathrm{Hach}^{\circledR}$ DR 2500 spectrophotometer. Data was collected three times per day, up to $35 \mathrm{~h}$. Absorbance values were compared between different assays. Measurements were taken with Odyssey Hach DR-2500 spectrophotometer at $600 \mathrm{~nm}$ wavelength. The tubes were left without agitation for $10 \mathrm{~min}$ before the start of each analysis. Tubes were not abruptly moved before being placed into the spectrophotometer to avoid any turbulence of the liquid, which could alter the absorbance results.

\subsubsection{Toxicity data analysis}

The colorimetric data from DCPIP $_{\text {ox }}$ discoloration has usually been considered analogous biodegradation data in microbial communities. Hanson et al. (1993), for example, initiated the trend of DCPIP applications in the monitoring of crude oil degradation by bacteria. However, we argue that experiments by Hanson et al. (1993) did not determine the biodegradability of compounds directly, but the amount of inhibition of microbial activity in different compounds. Many other legacy experiments using DCPIP followed this biodegradation correlation since then, including the recent studies by Varjani and Upasani (2016) and Souza et al. (2016). However, redox assays are in fact a direct measure of toxicity, instead of biodegradation. The electron transference in redox reactions drives the DCPIP molecular conformation changes and consequently color emission. Such redox reactions are not solely associated to a unique substrate consumption, but to the whole cell metabolism. Thus, what DCPIP actually measure is the microbial metabolic response to a given substrate and/or environment in which it is exposed instead of biodegradation.

The legacy DCPIP methodology is more properly described as a toxicity evaluation tool. Therefore, we exploited the parameters in discoloration kinetics to propose a new score system and assess the effect of AFFF to soil microbiota. Our research proposes the establishment and standardization of the DCPIP as a toxicity indicator. Models applied to the colorimetric dataset can lead to a better understanding of AFFF toxicity, thereby quickly sorting the level of toxicity of each concentration.

The colorimetric dataset was presented as $\mathrm{DCPIP}_{\mathrm{ox}}$ discoloration values as the oxidized form decreased though biodegradation. The depletion profile yielded by this process fitted to a sigmoidal non-linear model. The parameters were adjusted to describe our colorimetric data output (Eq. (3)). The model provided the toxicity level for the microbiota within each assay with the $S$ parameter.

$\mathrm{D}=\mathrm{D}_{\max }+\left[\left(\mathrm{D}_{\min }-\mathrm{D}_{\max }\right) /\left(1+10^{(\log \mathrm{D} 50-\mathrm{t}) \mathrm{S}}\right)\right]$

where: $\mathrm{D}=\mathrm{DCPIP}_{\mathrm{ox}}$ concentration, $\mathrm{D}_{\max }=$ initial $\mathrm{DCPIP}_{\mathrm{ox}}$ concentration, $\mathrm{D}_{\min }=$ minimum detectable $\mathrm{DCPIP}_{\mathrm{ox}}$ concentration, $\mathrm{D} 50=$ relative microbial response, $\mathrm{r}=$ toxicity score for a specific substrate and environment, $\mathrm{t}=$ time

The parameters of the sigmoidal equations were useful to obtain physiological activity data for each AFFF dilution. The D50 and $S$ kinetic parameters corresponded to units of time, where D50 defines the time when amplitude has reached its half-way point, i.e., when half of the DCPIP red $_{\text {has }}$ heen oxidized. The toxicity score $\mathrm{S}$ was defined as the time and concentration relationship for the parameter to go from maximum to minimum absorbance, which is translated as the toxicity intensity when soil microbiota is exposed to AFFF.

\section{Results and discussions}

\subsection{Toxicity of AFFF dilutions}

No seeds germinated in bioassays containing pure retail AFFF. The original $6 \%$ concentration and the $3 \%$ dilution did not result in germination in any of the triplicates. In $1 \%$ dilutions, there was an average germination of $3.4 \%$ germination of lettuce seeds and $1.7 \%$ of arugula seeds. The commercially available AFFF is labeled as a non-toxic product, however the phyto-toxicity results found in this study contradicts such statement.

The original formulation of AFFF is quite concentrated and disrupted seed germination environment. The acidity and the organic components of AFFF may have inhibited the germination of the test organisms. The fluorinated compounds are considered inert due to the number of $\mathrm{C}$-F bonds in their molecule, however they must have been another inhibiting factor. Fortunately, environmental samples contaminated with AFFF rarely contain concentrations as high as the ones tested. Contaminated environmental samples are usually close to the maximum $15.0 \mathrm{gL}^{-1}$ concentration allowed by USEPA (2009a).

\subsection{Toxicity of AFFF contaminated soil}

After $120 \mathrm{~h}$ of incubation, we observed that there was no germination in $\mathrm{ZnSO}_{4}$ control assays. Positive controls presented over $90 \%$ germination rates, meaning viable and healthy seeds. The germination percentages though time under different AFFF concentrations is shown in Fig. 1. The darker areas correspond to the lowest germination rates. 


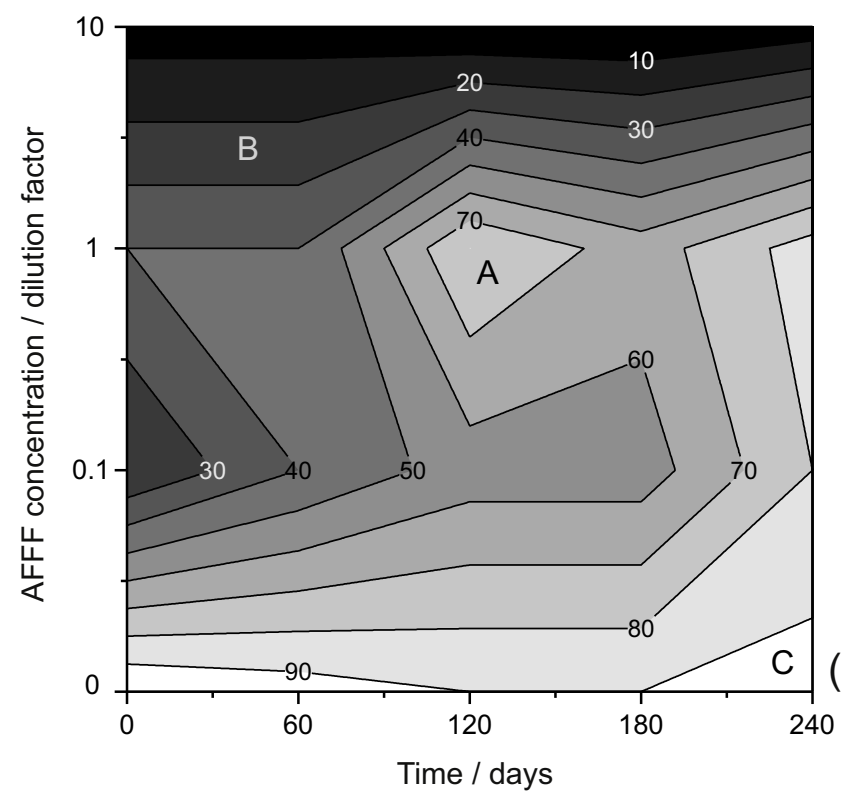

\section{Germination \%}

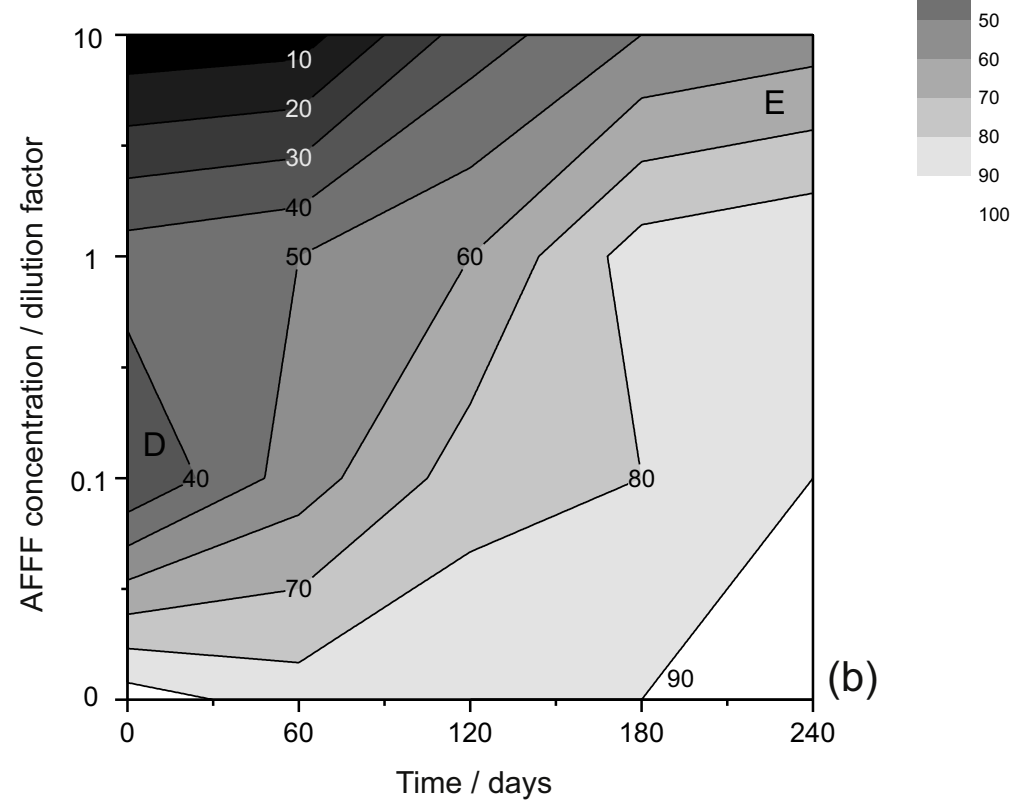

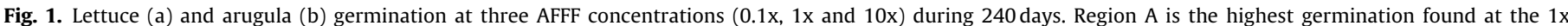

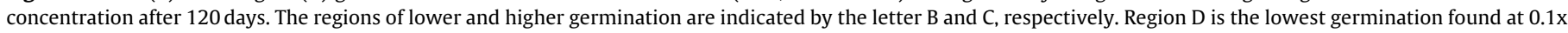
concentration. Region E shows the low sensitivity of arugula to AFFF.

The germination of seeds that were not exposed to AFFF in CO, C60, C120, C180 and C240 reached the highest values between 90 and 100\%. This can be observed throughout the lower region of both response surfaces in Fig. 1. The most toxic substrate, on the other hand, is seen on the upper part of the response surface plot, corresponding to the highest concentrations of AFFF (10x). Both arugula and lettuce had their lowest germination rates at $10 \mathrm{x}$ concentration even after 240 days. Lettuce was noticeably more sensitive to AFFF exposure than arugula. The lettuce sensitivity is especially noticeable in the region B (upper left corner) from Fig. 1a, where germination rates were inferior to $30 \%$ during the initial measurement time and after 60 days of natural attenuation (F0-10x and F60-10x). Arugula seeds, on the other hand, showed an increased germination (and decreased toxicity) at 10x concentrations in Fig. 1b. Even higher concentrations of AFFF allowed germination in the 30-50\% range at late attenuation time according to the region E (Fig. 1b), whereas lettuce seed were still germinating between the $0-20 \%$ range. Therefore, arugula seeds are less sensitive indicator of AFFF toxicity.

It is possible that biodegradation of non-fluorinated components of the formulation of AFFF may have contributed to the lower toxicity though time, resulting in increased germination after 120 days. The response surface indicates with the expansion of light gray tones on the right side of the chart a clear tendency to increase in germination. Thus, the simulated environment became more favorable to the growth of the seeds after 240 days. The Fig. 1 plot confirms visually that natural attenuation reduced the toxicity of AFFF especially at $1 \mathrm{x}$ and $0.1 \mathrm{x}$ concentrations.

According to Fig. 1, the germination was not exclusively a function of AFFF concentration ( $y$ ) or time (x) independently. The entire lettuce and arugula response surface approached a nonlinear correlation with time and germination, wherein the AFFF concentration 
affected sprouting. In other words, the response surface has shown interference between AFFF concentration (y) and germination ( $\mathrm{z}$ ) through time $(\mathrm{x})$.

Even though we observed that AFFF had a progressively low toxicity, there are some specificities that need to be highlighted. The region A in the lettuce response surface (Fig. 1a), for example, shows an interesting change in the expected pattern. An increase in germination occurred, specifically, around 120 days at $1 \mathrm{x}$ concentration (assay F120 at 1x concentration). Although these soil samples have not been chemically analyzed, it is likely that variants of secondary metabolites due to the biodegradation of AFFF have appeared throughout the process at that specific time. Those compounds that appeared only at the intermediate times (120 days) at $1 \mathrm{x}$ concentration are therefore less toxic and favored germination, whereas further AFFF degradation steps retained growth inhibition properties.

The natural attenuation is important to decrease toxicity of AFFF to such seeds. However, the seasonality factor must also be taken into account. We examined for any long-term correlation that may have disturbed germination. Despite the slightly higher germina-
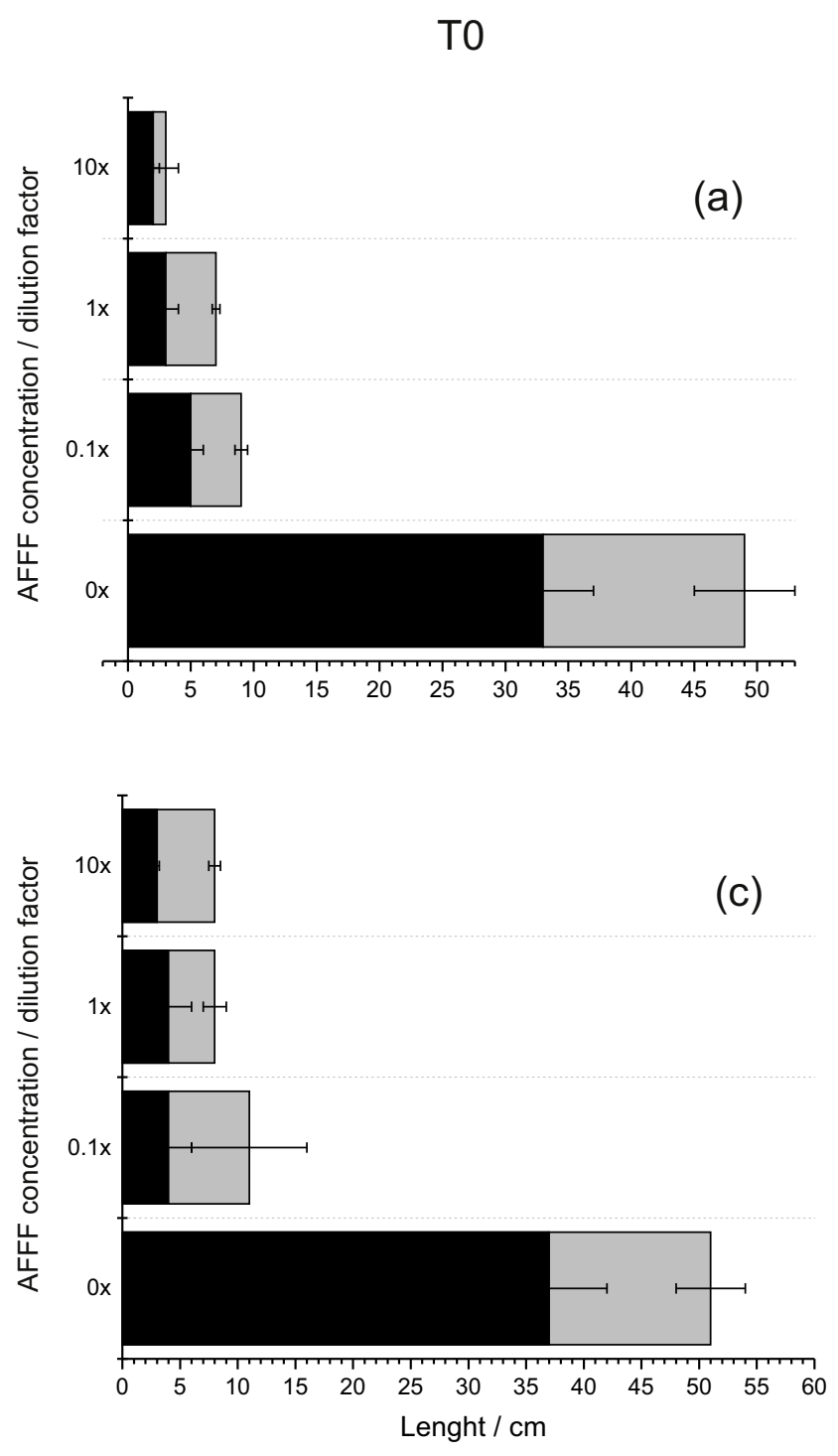

tion observed in region $\mathrm{C}$ of Fig. 1a, all datasets underwent Tukey test analysis to clarify whether the variation in germination rates between assays containing AFFF were significantly different from a seasonal variation. There was a significant difference between all assays compared to controls. Thus, the seasonal variation of soil conditions was not the factor responsible for increase in germination, but the difference of AFFF concentration between treatments instead.

Vegetable tissue growth also indicated the toxicity of the compounds before and after natural attenuation (Fig. 2).

As expected, soil controls without any PFC promoted higher vegetable tissue growth. Interestingly, a slightly higher average growth is found in lettuce at $1 \mathrm{x}$ concentrations rather than $0.1 \mathrm{x}$. Once more we observed the intermediate concentration (1x) interference with toxicity pattern in lettuce assays, where a decreased growth inhibition takes place at $1 \mathrm{x}$ concentration (Fig. 2d). Even though the phyto-toxicity of AFFF to lettuce and arugula was found to be high (increasing as a function of the concentration and decreasing as a function of exposure time to the environment), such very specific concentrations where non-inhibiting intermediates are
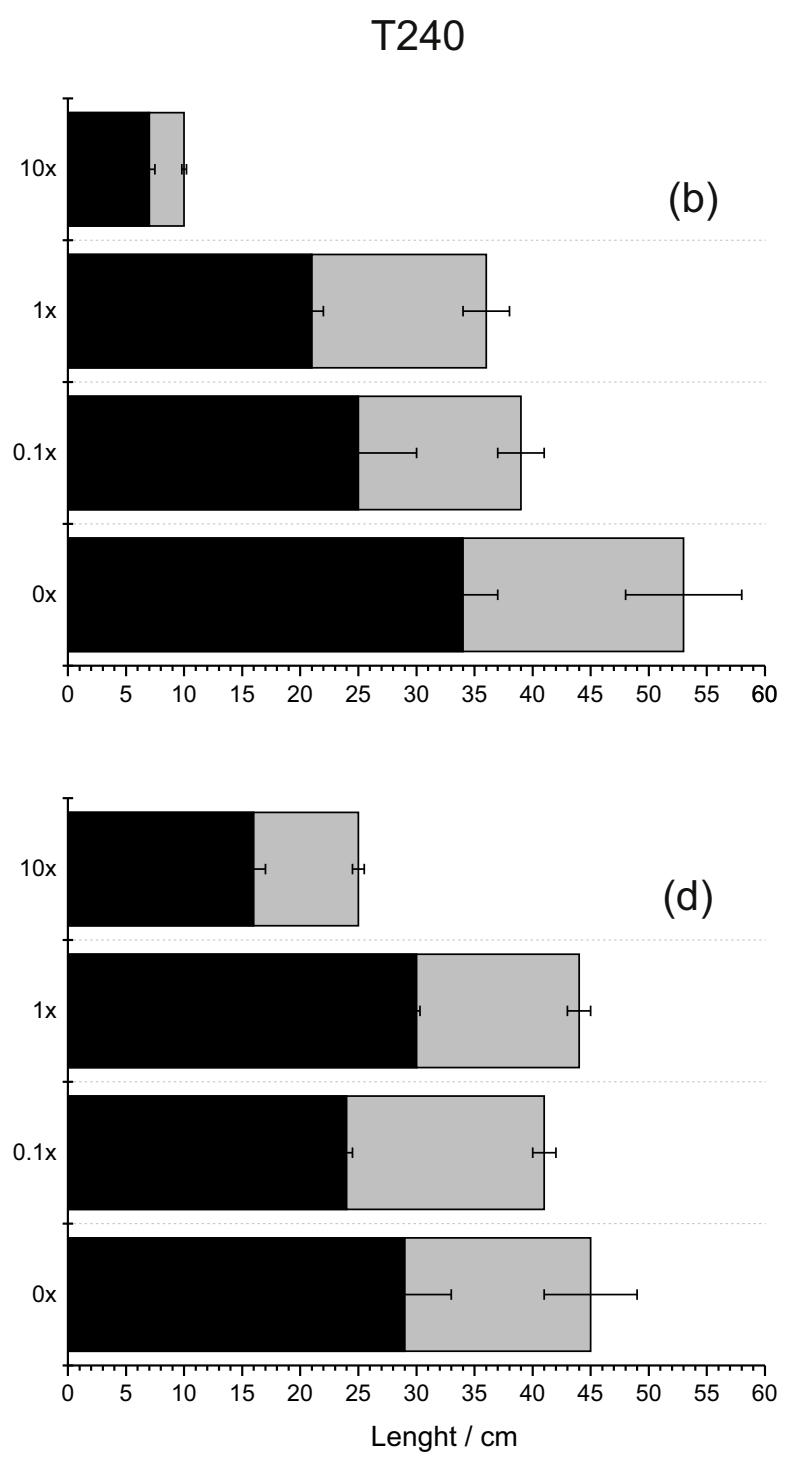

Hipocotyl Root

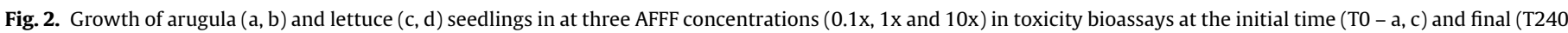
$-\mathrm{b}, \mathrm{d})$. 
formed may mislead other studies with non-time-based monitoring techniques. Therefore, it is imperative to consider the toxicity of PFCs in soil as a dynamic process where biodegradation and natural attenuation are key processes.

Arugula (Fig. 2a and b), however, followed a straightforward toxicity pattern. Higher AFFF concentrations yielded less arugula growth whereas lower AFFF concentrations allowed more cells to develop. The arugula seedlings were also much less sensitive to AFFF, as they were able to outgrow to lettuce seeds by $17.90 \%$ on average by the end of 240 days. Therefore, arugula seeds are not recommended to evaluate long-term AFFF toxicity during natural attenuation processes. Except for the low sensitivity after natural attenuation in arugula, both bioassays followed a similar growth pattern. The increase in germination and tissue growth over time caused AFFF toxicity was less pronounced in arugula seeds, hence lettuce is a better toxicity indicator for AFFF phyto-toxicity.

Lettuce and arugula tissue elongation data confirmed the influence of biodegradation time in reducing the toxicity of AFFF. The response surface in Fig. 1 has indicated higher germination representing higher germination (from $70 \%$ and upwards) on the right side of the chart. The seedling growth length reinforces that after 240 days a vegetable tissue growth is more likely to intensify. In general, the medium becomes more favorable to seeds growth after natural attenuation, but more sensitive organisms may not grow as much as the control assays.

There are many references that support low toxicity of nonfluorinated components of AFFF, such as butyl carbitol (Staples et al., 1998; Johansson, 1998). The toxicity of many perfluorinated compounds to plants, on the other hand, is not very well known. Studies report that these persistent PFCs are widely distributed in
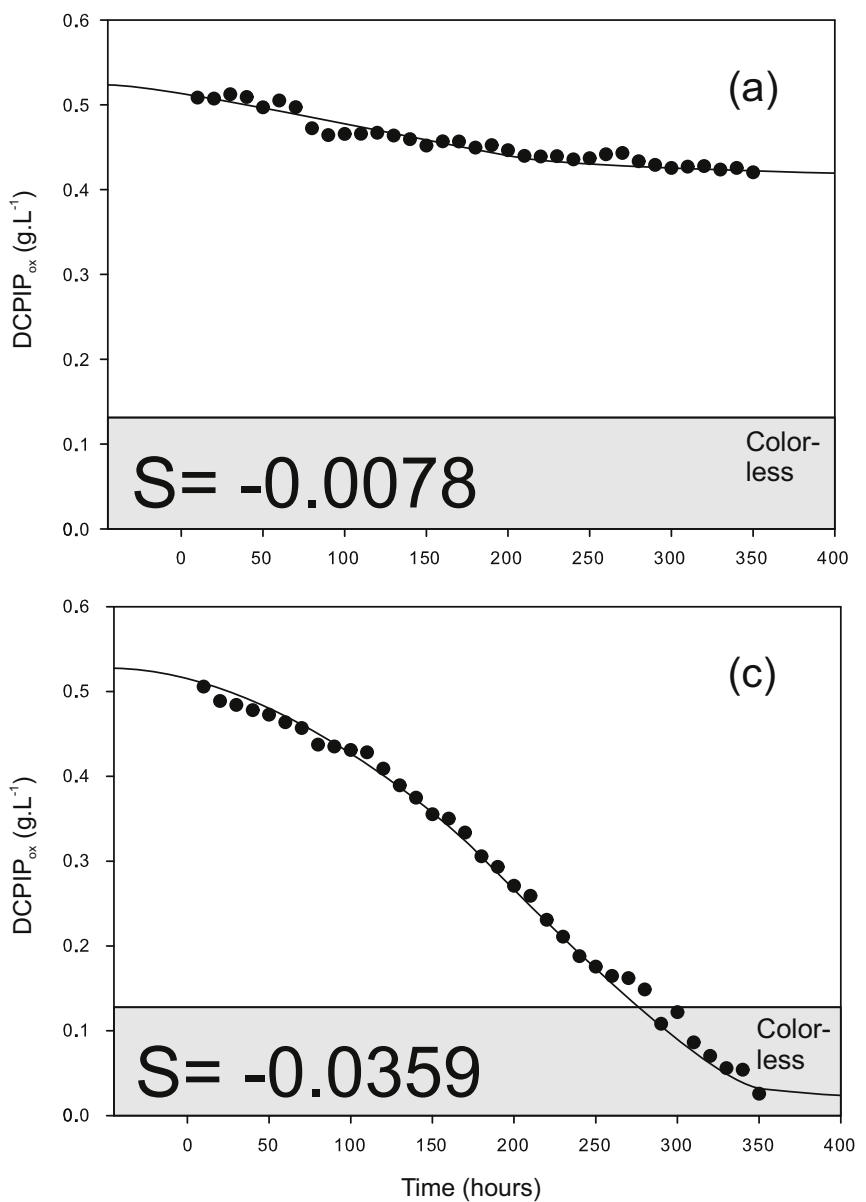

animals. It is known that exposure to them results in hepatotoxicity, development problems, immunotoxicity, adverse hormonal effects and potential carcinogen in animals (Clarke et al., 2010; Hölzer et al., 2008). Based on such previous toxicity studies in other organisms, it is safe to associate the toxicity observed in our bioassays to the presence of perfluorinated compounds. Still, there are no other reports of AFFF phyto-toxicity assays with arugula or lettuce that allow direct comparison to the results obtained in this study. The literature lacks firefighting foams related toxicity bioassays on plants. The hereby presented results comprehend an initial effort towards building a better comprehension of the AFFF effects in plants. Moreover, few studies on PFC toxicity analyze individual components and metabolites, since both the formulation as the secondary metabolites of biotransformation of PFCs are mostly unidentified. Thus, more in depth studies are encouraged to define the influence of AFFF in the development of many other test organisms.

The data obtained in toxicity bioassays, particularly shown in Fig. 1 indicate that, despite the initial toxicity of AFFF released into the environment either as arugula and lettuce have grown in contaminated soil. There is a major concern about the bioaccumulation of these compounds in the food chain, including in plants (Van Asselt et al., 2011). Although the arugula and lettuce growth may intuitively appear as an indication that the soil is safe for plants development due to decreased toxicity after 240 days, that does not mean that low toxicity is associated with the biodegradation of perfluorinated compounds. The butyl carbitol portion of AFFF formulation is the most biodegradable portion, whereas molecules containing multiple C-F bonds are considered biologically inert for these organisms. According to Filipovic et al. (2015), PFCs can enter
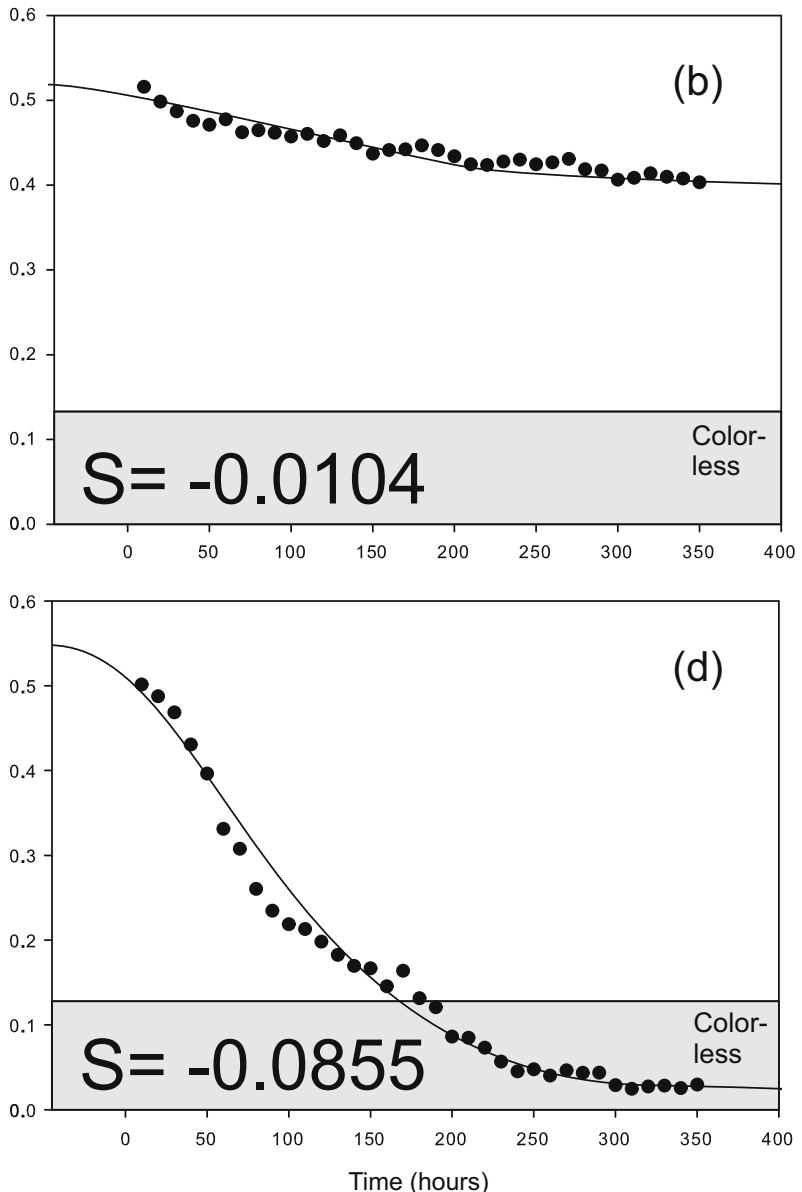

Fig. 3. DCPIP $_{\text {ox }}$ concentration in AFF control - C2 (a), $1000 \mu \mathrm{L}$ AFFF - B1 (b), $100 \mu \mathrm{L}-\mathrm{B} 2$ (c) and $10 \mu \mathrm{L}$ AFFF - B3 (d) assays. 
Table 3

Parameters set to discoloration model of DCPIP

\begin{tabular}{llll}
\hline Assay ID & Score $\left(\mathrm{g} \mathrm{L}^{-1} \mathrm{~h}^{-1}\right)$ & $\operatorname{DminT}(\mathrm{h})$ & $\mathrm{R}^{2}$ \\
\hline C0 & -0.0068 & - & 0.9932 \\
C1 & -0.0070 & - & 0.9928 \\
C2 & -0.0078 & - & 0.9956 \\
B1 & -0.0104 & - & 0.9942 \\
B2 & -0.0359 & 28.03 & 0.9939 \\
B3 & -0.0855 & 16.89 & 0.9968 \\
\hline
\end{tabular}

the tissues of plants during growth and accumulate in food chain for up to 45 years. This is potentially harmful, as fluorinated organic compounds are known for a decade to have bioaccumulative properties as well as toxicity to organisms, including mammals (Schultz et al., 2004).

We propose that AFFF germination and growth after 240 days, as observed in this study, may result in the PFCs entrance into the food-chain. The final germination rates (Fig. 1) can be related to PFC bioaccumulative potential. The main group of PFCs include PFOS, and has been frequently detected in food products at high concentrations (FSA, 2009). The route of human exposure to PFAS, however, has not yet been characterized (Halldorsson et al., 2008), but may as well come from the consumption of agricultural products. Although non-food sources, as even dust particles, may contain PFCs (and taken into consideration in Figure 85), the intake through food is the cause of $98 \%$ of human exposure the fluorocarbon (Fromme et al., 2009; Wilhelm et al., 2009). A recent study by Lorber et al. (2015) found up to $19 \mathrm{ng} \mathrm{mL}^{1}$ of perfluorinated compounds in human blood plasma.

Therefore, considering all potentially harmful substances reported by other authors, combined with our phyto-toxicity results, we encourage further studies on the bioaccumulation and toxicity of AFFF formulations to plants. For this reason, we also alert to the lack of strict legislation and control over the use, sale and disposal of AFFFs near cultivation areas.

\subsubsection{Soil microbiota inhibition}

The time required to complete decolorization varied among different concentrations of AFFF. Our colorimetric assays allowed the quantification of AFFF toxicity to soil microbiota (Fig. 3). The basic principle behind this technique is that the faster the curve reaches lower values of DCPIP ${ }_{\text {ox }}$, the faster microbial metabolism occurs.

The B2 and B3 assays were the only ones whose discoloration was complete. High AFFF concentrations in a soil sample may compromise microbial activity, thus decreasing the indicator color changes, especially when low amounts of AFFF is present.

Table 3 shows the expected parameters obtained from the adjustment model to the data of both methods. The most important parameter to be considered is S since this parameter can be arbitrarily employed towards toxicity rating in any given environment.

The equation parameters were adapted to the model when measuring absorbance values from each assay with differential AFFF concentration. The $S$ parameter varies approximately between -0.001 (more toxic) and -0.099 (less toxic) and allowed us to propose a quantifiable toxicity score from DCPIP color. The $S$ parameter analysis brings an interesting information on the toxicity of AFFF. It is expected to notice that the more diluted the sample is, the less toxic compound to soil microbiota. Thus, the highest dilution (Fig. 1d) presented the lowest level of toxicity, followed by B2, B1, and $\mathrm{C} 2$ assay.

The rates varied between the different substances. Even without the occurrence of full discoloration until the final measurement at $35 \mathrm{~h}$, we could precisely estimate the toxicity for each tested concentration. The parameters extrapolated by the model in adjusting allowed the prediction of toxicity even without complete discoloration DCPIP. Still, we observed from pilot assays that the predictability of dataset fitting to the equation is only minimally acceptable with a regular collection of data until at least $30 \mathrm{~h}$ into the process. With less than $30 \mathrm{~h}$ of periodically collected absorbance date, it is impossible to estimate relative toxicity with precision and consistency from the data set.

Overall, the addition of higher concentrations of AFFF caused less discoloration at all assays. PFCs would be responsible for inhibition of microbial activity in the assays as much as the observed phyto-toxicity in arugula and lettuce. Despite the positively obvious result, the curve fitting analysis should aid future regulations to define limits of AFFF and may as well be applied to any other pollutants released in soil.

\section{Conclusions}

The phyto-toxicity of AFFF to lettuce and arugula was found to be high, increasing as a function of the concentration and decreasing as a function of exposure time to the environment. Also, the low phyto-toxicity after 240 days and the potential for bioaccumulation in plants increases the persistence issues related to these compounds. The combination of two different methods of monitoring toxicity widened the current knowledge on the environmental release profile of AFFF. The assays allowed the monitoring of AFFF during long-term exposition to plants as well as their immediate effects on the same soil microbiota. While evaluating the compounds through natural attenuation, some possible biodegradation metabolites may have appeared and interfered with environmental safety at very specific concentrations.

Even though short-term experiments toxicity assays, such as the colorimetric assays, are usually not indicated towards monitoring of toxicity in degradation by-products, they allowed the precise modelling and quantification of AFFF effects on the soil microbiota. Phyto-toxicity assays further expanded the AFFF environmental safety assessment since they are operated on a much longer period, observing the appearance and disappearance of certain metabolites with an impact on toxicity. We observed that both techniques describe the AFFF environmental release scenario not only with a long-term decrease in toxicity, but also with an actual retardation of microbial metabolism in soil. Future studies are necessary to determine via further chemical analysis in plant tissues which bioconcentrated compounds result from AFFF metabolism in the environment. As plants were observed capable of growth in AFFF contaminated environment after 240 days, bioaccumulation of PFCs in the both test organisms (arugula and lettuce) is a likely assumption.

\section{Conflict of interest}

Nothing to disclose.

\section{Acknowledgments}

We gratefully acknowledge the thoughtful review and important insights provided by Sarah Mae Wachlin and Brent Perumal, providing comments on the manuscript in its entirety and working with the authors as we made revisions to the text. We alone, of course, take responsibility for the final text and any errors that appear therein.

Our research group acknowledges CAPES (Coordenação de Aperfeiçoamento de Pessoal de Nível Superior), CNPq (Conselho Nacional de Desenvolvimento Científico e Tecnológico), FUNDUNESP (Fundação para o Desenvolvimento da UNESP), PRHANP/MCT (Programa de Formação de Recursos Humanos em Geologia do Petróleo e Ciências Ambientais Aplicadas ao Setor de Petróleo e Gás) and UNESP (Universidade Estadual Paulista "Julio 
de Mesquita Filho") The financial support. funding sources had no involvement then this should be stated in the study design; in the collection, analysis and interpretation of data; in the writing of the report; and in the decision to submit the article for publication.

\section{References}

Alm R.R., Stern R.M., 1992. Aqueous Film-Forming Foamable Solution Useful as Fire Extinguishing Concentrate. U.S. Patent 5085786.

Ayers, R.S., Westcot, D.W., 1999. A qualidade da água na agricultura, Thesis. UFPB, Campina Grande.

Buck, R.C., Franklin, J., Berger, U., Conder, J.M., Cousins, I.T., De Voogt, P., Jensen, A.A., Kannan, K., Mabury, S.A., Van Leeuwen, S.P., 2011. Perfluoroalkyl and polyfluoroalkyl substances in the environment: terminology classification, and origins. Integr. Environ. Assess. Manag. 7, 513-541.

Clarke, B.O., Smith, S.R., 2011. Review of emerging organic contaminants in biosolids and assessment of international research priorities for the agricultural use of biosolids. Environ. Int. 37, 226-247.

Clarke, D.B., Bailey, V.A., Routledge, A., Lloyd, A.S., Hird, S., Mortimer, D.N., 2010. Dietary intake estimate for perfluorooctanesulphonic acid (PFOS) and other perfluorocompounds (PFCS) in UK retail foods following determination using standard addition LC-MS/MS. Food Addit. Contam. Part A Chem. 27, 530-545.

Dinglasan-Panlilio, M.J.A., Mabury, S.A., 2006. Significant residual fluorinated alcohols present in various fluorinated materials. Environ. Sci. Technol. 40, 1447-1453.

EECHA (European Chemical Agency), 2013. Candidate List of Substances of Very High Concern for Authorization, Available: http://echa.europa.eu/web/guest/ candidate-list-table. Accessed: June 2016.

Ellis, D.A., Martin, J.W., Desilva, A.O., Mabury, S.A., Hurley, M.D., Sulbaekandersen, M.P., Wallington, T.J., 2004. Degradation of fluorotelomer alcohols: a likely atmospheric source of perfluorinated carboxylic acids. Environ. Sci. Technol. 38, 3316-3321.

FSA (Food Standards Agency), 2009. Survey of Fluorinated Chemicals in Food. FSA, Available: www.food.gov.uk/multimedia/pdfs/fsis0509.pdf. Acessed: November 7.

Falk R.A., 1978. Aqueous Wetting and Film Forming Compositions. U.S. Patent 4090967.

Filipovic, M., Woldegiorgis, A., Norström, K., Bibi, M., Lindberg, M., Österås, A., 2015. Historical usage of aqueous film forming foam: a case study of the widespread distribution of perfluoroalkyl acids from a military airport to groundwater, lakes, soils and fish. Chemosphere 129, 39-45.

Frömel, T., Knepper, T.P., 2010. Biodegradation of fluorinated alkyl substances. Rev. Environ. Contam. Toxicol. 208, 161-177.

Fromme, H., Tittlemier, S.A., Völkel, W., Wilhelm, M., Twardella, D., 2009. Perfluorinated compounds -exposure assessment for the general population in western countries. Int. J. Hyg. Environ. Health 212, 239-270.

Giesy, J.P., Kannan, K., 2001. Global distribution of perfluorooctane sulfonate in wildlife. Environ. Sci. Technol. 35, 1339-1342.

Giesy, J.P., Kannan, K., 2002. Perfluorochemical surfactants in the environment. Environ. Sci. Technol. 36, 146-152.

Hölzer, J., Midasch, O., Rauchfuss, K., Kraft, M., Reupert, R., Angerer, J., Kleeschulte, P., Marschall, N., Wilhelm, M., 2008. Biomonitoring of perfluorinated compounds in children and adults exposed to perfluorooctanoate contaminated drinking water. Environ. Health Persp. 116, 651-657.

Hagner, M., Penttinen, O.P., Pasanen, T., Tiilikkala, K., Setälä, H., 2010. Acute toxicity of birch tar oil on aquatic organisms. Agric. Food Sci. 19, 24-32.

Halldorsson, T.I., Fei, C., Olsen, J., Lipworth, L., Mclaughlin, J.K., Olsen, S.F., 2008. Dietary predictors of perfluorinated chemicals: a study from the Danish National Birth Cohort. Environ. Sci. Technol. 42, 8971-8977.

Hanson, K.G., Desai, J.D., Desai, A.J., 1993. A rapid and simple screening technique for potential crude oil degrading microorganisms. Biotechnol. Tech. 7, 745-748.

Higgins, C.P., Field, J.A., Criddle, C.S., Luthy, R.G., 2005. Quantitative determination of perfluorochemicals in sediments and domestic sludge. Environ. Sci. Technol. 39, 3946-3956.

Houtz, E.F., Sedlak, D.L., 2012. Oxidative conversion as a means of detecting precursors to perfluoroalkyl acids in urban runoff. Environ. Sci. Technol. 46, 9342-9349.

Hudlicky, M., Pavlath, A.E., 1995. Chemistry of Organic Fluorine Compounds II: A Critical Review. American Chemical Society, Washington.

Johansson, G., 1998. Aspects of biological monitoring of exposure to glycol ethers. Toxicol. Lett. 43, 5-21.

Key, B.D., Howell, R.D., Criddle, C.S., 1997. Fluorinated organics in the biosphere. Environ. Sci. Technol. 31, 2445-2454.

Kim, S.K., Kannan, K., 2007. Perfluorinated acids in air, rain, snow, surface runoff, and lakes: relative importance of pathways to contamination of urban lakes. Environ. Sci. Technol. 41, 8328-8334.

Kissa, E., 1994. Fluorinated Surfactants: Synthesis, Properties and Applications, first ed. Marcel Dekker, New York.
Labouriau, L.G., Agudo, M., 1987. On the physiology of seed germination in Salvia hispanica L.I. Temperature effects. An. Acad. Bras. Ciênc. 59, 37-56.

Lladó, S., Solanas, A.M., De Lapuente, J., Borràs, M., Viñas, M., 2012. A diversified approach to evaluate biostimulation and bioaugmentation strategies for heavy-oil-contaminated soil. Sci. Total Environ. 435, 262-269.

Lorber, M., Eaglesham, G.E., Hobson, P., Toms, L.M.L., J.F. Mueller, J.F., Thompson, J.S., 2015. The effect of ongoing blood loss on human serum concentrations of perfluorinated acids. Chemosphere 118, 170-177.

Montagnolli, R.N., Lopes, P.R.M., Bidoia, E.D., 2015. Screening the toxicity and biodegradability of petroleum hydrocarbons by a rapid colorimetric method. Arch. Environ. Contam. Toxicol. 68, 342-353.

Moody, C., Field, J., 2000. Perfluorinated surfactants and the environmental implications of their use in fire-fighting foams. Environ. Sci. Technol. 34, 3864-3870.

Moody, C.A., Hebert, G.N., Strauss, S.H., Field, J.A., 2003. Occurrence and persistence of perfluorooctanesulfonate and other perfluorinated surfactants in groundwater at a fire-training area at Wurtsmith Air Force Base Michigan, USA. J. Environ. Monit. 5, 341-345.

Morales, C.G., 2004. Ensayos Toxicológicos y Métodos de Evaluación de Calidad de Agua: estandarización, intercalibración, resultados y aplicaciones, first edition. IMTA, Ciudad de México.

Murakami, M., Imamura, E., Shinohara, H., Kiri, K., Muramatsu, Y., Harada, A. Takada, H., 2008. Occurrence and sources of perfluorinated surfactants in rivers in Japan. Environ. Sci. Technol. 42, 6566-6572.

Nassif, S.M.L., Vieira, I.G., Fernandes, G.D., 1998. Informativo Sementes do Instituto de Pesquisas e Estudos Florestais, Thesis. Escola Superior de Agricultura Luís de Queiroz (ESALQ). Universidade de São Paulo (USP) Piracicaba.

Rayne, S., Forest, K., 2009. Perfluoroalkyl sulfonic and carboxylic acids: a critical review of physicochemical properties, levels and patterns in waters and wastewaters, and treatment methods. J. Environ. Sci. Health A 44, 1145-1199.

Renka, R.J., 1984. Interpolation of Data on the Surface of a Sphere. ACM Trans. Math. Softw. 10, 417-436.

Sardqvist, S., 2002. Water and Other Extinguishing Agents, fourth ed. Swedish Rescue Services Agency Karlstad.

Schultz, M., Barofsky, D., Field, J., 2004. Fluorinated alkyl surfactants. Environ. Eng. Sci. 20, 487-501.

Sheppard, P., Adetutu, E., Makadia, T., Ball, A., 2011. Microbial community and ecotoxicity analysis of bioremediated: weathered hydrocarbon-contaminated soil. Soil Res, 49, 261-269.

Sinclair, E., Kannan, K., 2006. Mass Loading and fate of perfluoroalkyl surfactants in wastewater treatment plants. Environ. Sci. Technol. 40, 1408-1414.

Snedecor, G.W., 1962. Statistical Methods. Iowa State University Press, Iowa.

Sokal, R.R., Rohlf, F.J., 1995. Biometry: the Principles and Practice of Statistics in Biological Research, third ed. W. H Freeman and Co., New York.

Souza, M.M., Colla, T.S., Bücker, F., Ferrão, M.F., Huang, C.T., Andreazza, R., Camargo, F.A.O., Bento, F.M., 2016. Biodegradation potential of Serratiamarcescens for diesel/biodiesel blends. Int. Biodeter. Biodeg. 110, 141-146.

Staples, C.A., Boatman, R.J., Cano, M.L., 1998. Ethylene glycol ethers: an environmental risk assessment. Chemosphere 36, 1585-1613.

USEPA (U.S. Environmental Protection Agency), 2009a. Provisional Health Advisories for Perfluorooctanoic Acid (PFOA) and Perfluorooctane Sulfonate (PFOS). U.S. Environmental Protection Agency, Washington.

USEPA (U.S. Environmental Protection Agency), 2009b. Soil Screening Levels for Perfluorooctanoic Acid (PFOA) and Perfluorooctyl Sulfonate (PFOS). U.S Environmental Protection Agency, Washington.

Van Asselt, E.D., Rietra, R.P.JJ.., Römkens, P.F.A.M., Van Der Fels-Klerx, H.J., 2011. Perfluorooctane sulphonate (PFOS) throughout the food production chain. Food Chem. 128, 1-6.

Varjani, S.J., Upasani, V.N., 2016. Biodegradation of petroleum hydrocarbons by oleophilic strain of Pseudomonas aeruginosa NCIM 5514. Bioresour. Technol. $222,195-201$

Vestergren, R., Cousins, I.T., 2009. Tracking the pathways of human exposure to perfluorocarboxylates. Environ. Sci. Technol. 43, 5565-5575.

Wilhelm, M., Holzer, J., Dobler, L., Rauchfuss, K., Midasch, O., Kraft, M., 2009. Preliminary observations on perfluorinated compounds in plasma samples (1977-2004) of young German adults from an area with perfluorooctanoate-contaminated drinking water. Int. J. Hyg. Envir. Heal. 212, $142-145$

Yoshida, N., Hoashi, J., Morita, T., McNiven, S.J., Nakamura, H., Karube, I., 2001. Improvement of a mediator-type biochemical oxygen demand sensor for on-site measurement. J. Biotechnol. 88, 269-275.

Yu, J., Hu, J., Tanaka, S., Fujii, S., 2009. Perfluorooctane sulfonate (PFOS) and perfluorooctanoic acid (PFOA) in sewage treatment plants. Water Res. 43 $2399-2408$.

Yuan, F., 1998. Automatic drawing of equal quantity curve. Comput. Aided Chem. 1, $1-7$.

Zushi, Y., Masunaga, S., 2009. First-flush loads of perfluorinated compounds in stormwater runoff from Hayabuchi River basin, Japan served by separated sewerage system. Chemosphere 76, 833-840. 